\title{
Adaptación y validación de los cuestionarios de apoyo a la autonomía y estilo controlador a la educación física: relación con el feedback
}

\section{Adaptation and validation of autonomy support and controller style's scales in physical education: relationship with feed-back}

*Juan Antonio Moreno-Murcia, Elisa Huéscar, *José Antonio Andrés-Fabra \& *Francisco Sánchez-Latorre

Moreno-Murcia, J., Huéscar, E., Andrés-Fabra,J., \& Sánchez-Latorre, F. (2020). Adaptación y validación de los cuestionarios de apoyo a la autonomía y estilo controlador a la educación física: relación con el feedback. Revista Ciencias de la Actividad Física UCM, N²7(7), enero-junio, 1-16. DOI: http://doi.org/10.29035/rcaf.21.1.3

\section{RESUMEN}

El objetivo de este trabajo fue adaptar y validar la Escala de Apoyo a la Autonomía (EAA-EF) y la Escala de Estilo Controlador (EEC-EF) para evaluar la percepción del estudiante del estilo interpersonal docente en educación física y comprobar su relación con el feedback. El estudio 1, contempla el diseño y análisis factorial exploratorio de los instrumentos con una muestra de 272 estudiantes con una edad media de 11.05 años $(D T=0,76)$. En el estudio 2, los cuestionarios fueron administrados a 350 estudiantes con una edad media de 11 años $(D T=0,80)$. Tras el análisis factorial confirmatorio, de consistencia interna, estadísticos descriptivos, correlación de todas las variables y regresión lineal se confirma una estructura psicométrica apropiada para las escalas de apoyo a la autonomía y estilo controlador. Además, se observa cómo el apoyo a la autonomía es predicho positivamente por los feedbacks positivos y negativamente por el negativo. En conclusión, se trata de dos instrumentos válidos y fiables para evaluar la percepción del estudiante del estilo interpersonal del docente de educación física en el escenario de enseñanza-aprendizaje, siendo necesarios más estudios en este ámbito de conocimiento.

Palabras clave

Motivación autodeterminada, evaluación docente, instrumento de medida, apoyo a la autonomía, control, feedback. 
Moreno-Murcia, J., Huéscar, E., Andrés-Fabra,J., \& Sánchez-Latorre, F. (2020). Adaptación y validación de los cuestionarios de apoyo a la autonomía y estilo controlador a la educación física: relación con el feedback.

Revista Ciencias de la Actividad Física UCM, N²1(1), enero-junio, 1-16. DOI: http://doi.org/10.29035/rcaf.21.1.3

\section{ABSTRACT}

The objective of this study was to adapt and validate The Autonomy Support Scale (ASS-PE) and The Control Style Scale (CSS-PE) to evaluate teacher's interpersonal style in Physical Education. Study 1, contemplates the design and exploratory factor analysis of the instruments with a sample of 272 students with an average age of 11.05 years (SD = 0.76). In study 2 , the questionnaires were administered to 350 students with an average age of 11 years (SD $=0.80)$. After the confirmatory factor analysis, internal consistency and descriptive statistics, the correlation of all the variables and linear regression, an appropriate psychometric structure is confirmed for the support scales for autonomy and controlling style. In addition, it is observed how support for autonomy is predicted positively by positive feedbacks and negatively by negative feedback. The data obtained confirms an appropriate psychometric structure for ASS-PE and CSS-PE scales. In conclusion, it is a valid and reliable instrument for evaluating the interpersonal style of teacher 's physical education in the teaching-learning stage.

\section{Key words}

Self-determination motivation, teaching evaluation, measuring instrument, autonomy support, control, feed-back.

\section{Introducción}

A pesar de que en España la autonomía escolar ha estado sujeta a sucesivos cambios legislativos desde los años 90, en la última Ley Orgánica 8/2013, de 9 de diciembre para la mejora de la calidad educativa (LOMCE), se propone un mayor refuerzo al papel de la autonomía en los centros educativos, señalando como finalidad la mayor adecuación y aprovechamiento de los recursos asignados y el ajuste de la acción pedagógica a las necesidades de los estudiantes. Durante la última década, la comunidad científica, a partir de los resultados de los principales estudios internacionales (Euryce, 2008, PISA, 2012, TALIS, 2013) que apuntan que, a mayor autonomía escolar, mayor rendimiento del estudiante, está muy interesada en conocer las variables implicadas en este proceso.

En este sentido, el estudio de la autonomía escolar en relación a la intervención docente, es uno de los caminos que podría contribuir a comprender estas relaciones. Así pues, los entornos de aprendizaje constructivistas que posibilitan al estudiante aprender significativamente a través de su propio esfuerzo, está ganando fuerza frente a otros entornos de aprendizaje más tradicional basados, por ejemplo, en la reproducción y asimilación mecánica de contenidos (Oguz, 2013). Según este enfoque constructivista, uno de los principales objetivos de la educación, radica en promover la autonomía del estudiante (Öztürk, 2011). Vallerand (1997) señala que las consecuencias que permiten favorecer el comportamiento, la cognición y a afectividad pueden depender de factores sociales en los que se desenvuelve el estudiante (Teixeira \& Laranjeira, 2018), donde el estilo docente sería una variable fundamental. Concretamente, la forma en la que el estudiante percibe que la actuación docente le permite elegir, reducir la presión en su actuación y en el control externo de su conducta (Hagger, Chatzisarantis, Hein, Pihu, Soós \& Karsai, 2007, Standage, Duda, \& Ntoumanis, 2006) le posibilitará regular su comportamiento atendiendo a sus intereses (De Miguel, Marrero, Fumero, \& Carballeira, 2017). Los docentes que apoyan la autonomía de sus estudiantes tienen un estilo que se define por tener en cuenta sus perspectivas, pensamientos y sentimientos, motiván- 
doles a través del fomento de sus recursos motivacionales internos. En el otro extremo, un estilo docente controlador, se caracterizaría por exigir que los estudiantes tengan solamente la perspectiva que el docente propone, condicionando los pensamientos, sentimientos y acciones de sus estudiantes. Por ejemplo, los presionaría mediante un lenguaje directivo e impaciente a proceder como él impone motivándolos solamente con incentivos externos como las calificaciones (Reeve, 2009). Finalmente, ambas dimensiones, la que apoya a la autonomía del estudiante y la que la intenta controlar, son reflejo de una serie de comportamientos comunicativos verbales y no verbales que el docente manifiesta sobre la actuación del estudiante en clase conocido como feed- back (Koka \& Hein, 2005). Los estudios vienen demostrando su influencia sobre la calidad del proceso de enseñanza-aprendizaje (Valdivia, 2011, Moreno-Murcia, Huéscar, Peco, Alarcón, \& Cervelló, 2013) repercutiendo tanto en la actuación del docente (Ureña, Alarcón, \& Ureña, 2009) como en el aprendizaje y motivación de los estudiantes (Vallejo \& Plested, 2008).

Así pues, a partir de los postulados de la Teoría de la Autodeterminación (TAD, Ryan \& Deci, 2000) el apoyo a la autonomía se presenta como un factor fundamental que puede influir en la capacidad de los estudiantes para desarrollarse tanto en el ámbito personal como social. En este sentido, las clases de educación física (EF) suponen un escenario idóneo como generador de experiencias positivas como la instauración de hábitos saludables y duraderos. Estas experiencias pueden deberse en parte al apoyo que el docente de EF ejerza de manera significativa sobre la necesidad de autonomía del estudiante (Aibar, Julián, Murillo, GarcíaGonzález, Estrada, \& Bois, 2015, Lim \& Wang, 2009). No obstante, a pesar de que la TAD (Deci \& Ryan, 2000) también establece la relación entre la percepción de apoyo a la autonomía del estudiante con la práctica de actividad física y la motivación (Gallagher, Yancy, Swartout, Denissen, Kuhnel, \& Voils, 2012) incluso señalando que un estilo de apoyo a la autonomía del docente predice positivamente un mayor compromiso de los estudiantes hacia la práctica de actividad física (Haerens, Hirk, Cardon, De Bourdeaudhuij, \& Vansteenkiste, 2010). Sin embargo, no contamos con ningún instrumento de medida que nos ayude a establecer en clases de educación física el grado de apoyo a la autonomía y control que percibe el estudiante del estilo interpersonal docente.

A partir del trabajo de Standage et al. (2006) promover climas de aula que apoyen a la autonomía del estudiante contribuiría positivamente con las tres necesidades psicológicas básicas de la TAD (autonomía, competencia y relación con los demás) por lo que atender a los tres aspectos se presenta fundamental en su evaluación. No obstante, los estudios recientes (Abós, Sevil, Sanz, Aibar, \& García-González, 2016) que han precisado la evaluación del apoyo de autonomía del docente de EF suelen recurrir únicamente a la necesidad de autonomía, atendiendo al factor apoyo a la autonomía que contempla junto a las otras dos necesidades (competencia y relación) el Cuestionario de Apoyo a las Necesidades Psicológicas Básicas (CANPB, Sánchez-Oliva, Leo, Amado, Cuevas, \& García-Calvo, 2013). Dicho factor está compuesto de cuatro ítems (e.g., "Nos deja tomar decisiones..."). En esta línea, otro indicador más general sobre el grado de implicación e interés del docente con los estudiantes en clase lo proporciona la Teacher's Care, de Röder y Klein (2007), validada al contexto español por Moreno-Murcia, Ruíz, Silveira, \& Alias (2017). En cuanto a los climas de enseñanza-aprendizaje que contemplan el apoyo a la autonomía, la Learning Climate Questionnaire (LCQ) tiene una versión española adaptada a la EF (LCQ-EF) (GraneroGallegos, Baena-Extremera, Pérez, Quero, Ortíz-Camacho, \& Bravo-Amador, en prensa) 
Moreno-Murcia, J., Huéscar, E., Andrés-Fabra,J., \& Sánchez-Latorre, F. (2020). Adaptación y validación de los cuestionarios de apoyo a la autonomía y estilo controlador a la educación física: relación con el feedback.

Revista Ciencias de la Actividad Física UCM, N²1(1), enero-junio, 1-16. DOI: http://doi.org/10.29035/rcaf.21.1.3

del original de Williams \& Deci (1996) a través de 14 ítems y esta única dimensión.

Por su parte, respecto al estilo controlador, pese a que la investigación es más escasa que la dedicada al apoyo a la autonomía, resulta determinante su estudio por las consecuencias negativas demostradas sobre los discentes, como su relación positiva con la desmotivación del estudiante al verse frustradas las necesidades psicológicas básicas (Soenens, Park, Vansteenkiste, \& Mouratidis, 2012). Respecto a su medida, en el ámbito deportivo destaca la escala de la percepción del estilo interpersonal controlador del entrenador (CCBS, Bartholomew, Ntoumanis, \& Thøgersen-Ntoumani, 2010, Castillo, Tomás, Ntoumanis, Bartholomew, Duda, \& Balaguer, 2014), compuesta por 15 ítems divididos en cuatro subescalas (uso controlador de recompensas, atención condicional negativa, intimidación, excesivo control personal). En el ámbito de la Educación Superior, recientemente, la Escala de Medición del Estilo Controlador (Moreno-Murcia, Pintado, Huéscar, \& Marzo, 2018) unifica a partir de trabajos anteriores (Standage, Duda, \& Ntoumanis, 2005) en un solo constructo las necesidades psicológicas básicas simplificando su medida y dedicándolo a la etapa de estudios superiores. Para el apoyo a la autonomía, la Escala de Apoyo a la Autonomía (Moreno-Murcia, Huéscar, Pintado, \& Marzo, 2019) mide en un único factor el apoyo a la autonomía que perciben los estudiantes de sus docentes de Educación Superior. En la misma línea, la Escala de Estilo Controlador del Estudiante (ECE) (Huéscar, MorenoMurcia, \& Alías, 2017) focaliza en los estilos de interacción entre los estudiantes de Educación Superior cuando gestionan una tarea académica, y para la medida del estilo de apoyo a la autonomía entre estudiantes de Educación Superior, la Escala de Apoyo a la Autonomía del Estudiante (EAE, Huéscar \& Moreno-Murcia, 2017) ha sido recientemente diseñada y validada con estudiantes españoles. Además, en dicho estudio, el apoyo a la autonomía predecía positivamente la satisfacción con la vida de los estudiantes, mientras que el miedo a la evaluación negativa lo hacía en sentido negativo.

Tomando como referencia la TAD, y a partir de la importancia demostrada de los factores sociales (estilo de apoyo a la autonomía/ estilo controlador del docente) para los resultados motivacionales del estudiante (Vallerand, 1997), el objetivo de este estudio fue contribuir al diagnóstico de dichos patrones de comportamiento docente en Educación Física. Así pues, se planteó diseñar y validar dos instrumentos que evalúen el estilo de apoyo a la autonomía y el controlador en el docente en clases de EF, teniendo en cuenta no solamente la necesidad psicológica básica de autonomía (Sánchez- Oliva et al., 2013) sino también la de competencia y relación con los demás. Esta perspectiva ampliada, viene apoyada por los resultados de investigaciones basadas en la TAD que asumen que el apoyo a la autonomía es fundamental para satisfacer las necesidades psicológicas básicas, como la de Standage et al. (2006) que relacionan positivamente el apoyo a la autonomía con las tres necesidades psicológicas. Además, partiendo de los estudios previos descritos sobre la necesidad de que los docentes desarrollen climas no amenazantes a través de retos realistas y feedback positivo que desarrolle la motivación intrínseca (Koka \& Hein, 2003), se hipotetizó que la percepción de un feedback positivo (tanto verbal como no verbal) como referido al conocimiento del rendimiento del estudiante prediría positivamente el estilo de apoyo a la autonomía y en sentido negativo el estilo controlador. 


\section{Estudio 1}

\section{Método}

\section{Participantes}

La muestra estuvo compuesta por 272 estudiantes de educación física (142 chicos y 130 chicas) de $5^{\circ}$ y $6^{\circ}$ curso de Educación Secundaria Obligatoria (ESO), pertenecientes a varios centros escolares de una gran provincia española, con una edad media de 11,15 años $(D T=0,76)$. Los participantes fueron seleccionados de centros escolares mediante muestreo aleatorio por conglomerados (Azorín \& Sánchez-Crespo, 1986).

\section{Medidas e Instrumentos}

Apoyo a la autonomía. Se diseñó la Escala de Apoyo a la Autonomía en Educación Física (EAA-EF) que finalmente quedó compuesta por 13 ítems que a través de un único factor mide la necesidad de apoyo a la autonomía que perciben los estudiantes de sus docentes en clases de educación física. Los ítems (e.g. "Con sus explicaciones, nos ayuda a comprender para qué sirven las actividades que realizamos") estaban precedidos por la sentencia previa "En mis clases de educación física, mi profesor/a...". Se midió a través de una escala tipo Likert que va desde 1 (Seguro que no) a 5 (Seguro que si).

Estilo controlador. Se diseñó la Escala de Estilo Controlador en Educación Física (EEC-EF) compuesta por 9 ítems que miden el estilo controlador que perciben los estudiantes de sus docentes en clases de educación física. Los ítems (e.g. "Cuando no nos esforzamos en hacer las cosas como él dice, nos hace menos caso") estaban precedidos por la sentencia previa "En mis clases de educación física, mi profesor/a...". Se utilizó una escala tipo Likert cuyas opciones eran 1 (Seguro que no) a 5 (Seguro que si).

\section{Procedimiento}

Se llevó a cabo una revisión bibliográfica, para la elaboración de los ítems. Estos fueron revisados por expertos que puntuaron, en una escala Likert de cinco puntos sobre cada ítem de las escalas, la suficiencia, la claridad de lenguaje, la coherencia y su relevancia teórica. Las conclusiones que se sacaron con esta evaluación fueron encaminadas a reescribir algunos ítems. Posteriormente, se administró a un grupo de estudiantes ( $n=$ 22) para comprobar su comprensión. Fue entendida y no se realizaron cambios.

Se solicitó la autorización a los equipos directivos de los centros escogidos y a los padres/tutores de los mismos. Fueron unos 12 minutos de media el tiempo necesario para su cumplimentación. Los criterios de inclusión fueron ser estudiantes de $5^{\circ}$ y $6^{\circ}$ curso asistentes a las clases de educación física en los centros seleccionados, mientras que los criterios de exclusión fueron el no contestar correctamente los instrumentos de medida. El estudio obtuvo la aprobación del órgano evaluador de proyectos de la Universidad Miguel Hernández de Elche con el número de registro DPS.JMM.01.17.

\section{Análisis estadístico}

Se analizaron las propiedades psicométricas de las escalas de medida a través de un análisis factorial exploratorio. Se analizó la consistencia interna de cada factor mediante el coeficiente de Alfa de Cronbach. Los datos fueron analizados mediante el paquete estadístico SPSS 21.0 y AMOS 21.0. 
Moreno-Murcia, J., Huéscar, E., Andrés-Fabra,J., \& Sánchez-Latorre, F. (2020). Adaptación y validación de los cuestionarios de apoyo a la autonomía y estilo controlador a la educación física: relación con el feedback.

Revista Ciencias de la Actividad Física UCM, N²1(1), enero-junio, 1-16. DOI: http://doi.org/10.29035/rcaf.21.1.3

\section{Resultados}

\section{Análisis factorial exploratorio de la EAA-EF y EEC-EF}

Se analizaron sus propiedades psicométricas a través de un análisis factorial exploratorio de componentes principales con rotación varimax. Los resultados indicaron que los ítems de cada escala se agrupaban en un solo factor. El porcentaje de varianza total explicada fue del $61,65 \%$ para la EAA-EF y los pesos de saturación oscilaron de 0,54 a 0,89, y de $71,23 \%$ para la EEC-EF, oscilando las saturaciones de .51 a .90. La consistencia interna de las escalas fue de 0,71 para la EAA-EF y 0,77 para la EEC-EF.

\section{Estudio 2}

\section{Método}

\section{Participantes}

La muestra estuvo compuesta por 350 estudiantes de educación física (184 chicos y 166 chicas) de $5^{\circ}$ y $6^{\circ}$ curso de Educación Secundaria Obligatoria (ESO), pertenecientes a varios centros escolares de una gran provincia española, con una edad media de 11,05 años $(D T=.80)$. Los participantes fueron seleccionados de centros escolares mediante muesSánchez-Crespo, 1986).

\section{Medidas}

Apoyo a la autonomía. Se utilizó la escala descrita en el estudio 1. La consistencia interna fue de 0,77.

Estilo controlador. Se utilizó la escala descrita en el estudio 1. La consistencia interna obtuvo un valor de 0,71 para el estilo controlador.

Percepción del feedback. Se utilizó el Cuestionario de las percepciones del feedback del docente-revisado (PTF-R) de Koka y Hein

(2005), validado al contexto español por Huéscar y Moreno-Murcia (2012). Está formada por 14 ítems agrupados en 4 factores: feedback general positivo percibido (e.g. "Mi trabajo es con frecuencia animado/alentado por el profesor/a"), conocimiento del rendimiento percibido (e.g. "Cuando lo hago bien en educación física, el profesor/a lo confirma"), feedback no verbal positivo general (e.g. "El profesor/a sonríe cuando realizo una buena ejecución") y feedback no verbal negativo general (e.g. "El profesor/a sacude o mueve su cabeza cuando realizo una mala ejecución"). La sentencia previa fue "En mis clases de educación física...". Las respuestas fueron valoradas a través de una escala tipo Likert que oscilaba desde 1 (Falso) a 5 (Verdadero). La consistencia interna para el feedback general positivo percibido fue de 0,70, para el conocimiento del rendimiento percibido de 0,71, para el feedback no verbal positivo general de 0,76 y para el feedback no verbal negativo general de 0,69.

\section{Procedimiento}

Se siguió el mismo procedimiento recogido en el estudio 1.

\section{Análisis estadístico}

Fueron calculados los estadísticos descriptivos y se utilizó el coeficiente de Alfa de Cronbach para el cálculo de la consistencia interna. También se realizaron análisis de correlaciones. La escala se confirmó a través de un análisis factorial confirmatorio. Asimismo, se comprobó el poder predictivo del feedback general positivo percibido, el conocimiento del rendimiento percibido, el feedback no verbal positivo general y el feedback no verbal negativo general sobre el soporte de autonomía y el estilo controlador, a través de un análisis de regresión lineal múltiple por pasos. Los datos fueron analizados mediante el paquete estadístico SPSS 21.0 y AMOS 21.0. 
Análisis factorial confirmatorio de la escala de apoyo a la autonomía

Indicar que previamente se comprobó a través de un análisis factorial confirmatorio (CFA) con los 13 ítems iniciales que componían la escala, pero dos reactivos presentaron un bajo peso de regresión estandarizado. Se realizó un nuevo CFA basándonos en 11 medidas observadas. La validez del modelo de medición fue considerada a través de una serie de coeficientes fit, también Ilamados índices de bondad de ajuste: $\chi^{2}, \chi^{2} / d$.f., RMSEA, RSMR y los índices incrementales
(CFI, IFI y TLI). Fue utilizado el método de estimación de máxima verosimilitud junto con el procedimiento de bootstrapping, pues el coeficiente multivariado de Mardia fue 30.84, lo que indicaba falta de normalidad multivariada de los datos. Por ello, siguiendo a Finney \& DiStefano (2006) se utilizó el método robusto de estimación de máxima verosimilitud (Byrne, 2001). Los índices obtenidos fueron adecuados: $\chi^{2}(44, N=350)=$ 86,92, $p<.000, \chi^{2} /$ d.f. $=1,97, \mathrm{CFI}=0,93, \mathrm{IFI}=$ $0,93, \mathrm{TLI}=0,91, \mathrm{RSMR}=0,04, \mathrm{RMSEA}=0,05$ (Tabla 1).

\section{Tabla 1}

Parámetros Estimados de AFC para la Escala del Apoyo a la Autonomía en Educación Física (EAA-EF).

\section{M}

\section{DT Asimetría}

1. Con sus explicaciones, nos ayuda a comprender para qué sirven las actividades que realizamos.

2. Valora nuestras ideas y sugerencias y nos deja proponer cosas.

3. Nos explica por qué es importante realizar tareas.

4. Cuando tenemos dificultades para resolver una tarea, nos ayuda y nos apoya para que busquemos la solución nosotros mismos.

5. Ofrece diferentes formas de realizar una determinada tarea.

6. Permite que podamos decidir sobre lo que queremos aprender.

7. Si nos quejamos por algo nos escucha y atiende nuestras razones.

8. Nos permite resolver las tareas a nuestra manera.

9. Intenta que cada vez seamos más autónomos.

10. Nos deja el tiempo que necesitemos para aprender nuevos contenidos.

11. Responde nuestras preguntas de forma atenta y detallada, tanto individualmente como a toda la clase.

\begin{tabular}{|c|c|c|c|c|c|}
\hline 2,07 & 1,31 & $-1,87$ & 3,22 & 0,43 & 0,19 \\
\hline 1,67 & 1,12 & $-1,26$ & 0,58 & 0,57 & 0,33 \\
\hline 2,22 & 1,43 & $-1,07$ & 0,17 & 0,48 & 0,23 \\
\hline 2,25 & 1,49 & $-1,41$ & 1,08 & 0,57 & 0,32 \\
\hline 2,15 & 1,41 & $-0,79$ & $-0,42$ & 0,52 & 0,27 \\
\hline 2,22 & 1,47 & 0,05 & $-1,35$ & 0,47 & 0,22 \\
\hline 3,11 & 1,49 & $-0,96$ & $-0,51$ & 0,65 & 0,42 \\
\hline 2,71 & 1,52 & $-0,03$ & $-1,14$ & 0,31 & 0,09 \\
\hline 2,41 & 1,52 & $-0,67$ & $-0,66$ & 0,35 & 0,12 \\
\hline 2,07 & 1,31 & $-0,87$ & $-0,44$ & 0,43 & 0,19 \\
\hline 1,67 & 1,12 & $-1,61$ & $-1,34$ & 0,59 & 0,34 \\
\hline
\end{tabular}


Moreno-Murcia, J., Huéscar, E., Andrés-Fabra,J., \& Sánchez-Latorre, F. (2020). Adaptación y validación de los cuestionarios de apoyo a la autonomía y estilo controlador a la educación física: relación con el feedback.

Revista Ciencias de la Actividad Física UCM, N²1(1), enero-junio, 1-16. DOI: http://doi.org/10.29035/rcaf.21.1.3

\section{Análisis factorial confirmatorio de la es- cala de estilo controlador}

Se realizó un análisis factorial confirmatorio (CFA) basándonos en las nueve medidas observadas en el constructo estilo controlador. Debido a que el resultado del coeficiente multivariado de Mardia fue 16,34, lo que indicaba falta de normalidad multivariada de los datos, se utilizó el método de estimación de máxima verosimilitud junto con el procedimiento de bootstrapping. Los índices obtenidos fueron adecuados: $\chi^{2}(36, N=350)$ $=46,00, p<.01, \chi^{2} /$ d.f. $=1,76, \mathrm{CFI}=0,92, \mid \mathrm{FI}=$ $0,93, \mathrm{TLI}=0,90, \mathrm{RSMR}=0,04, \mathrm{RMSEA}=0,04$ (Tabla 2)

\section{Tabla 2}

Parámetros Estimados de AFC para la Escala Estilo Controlador en Educación Física (EECEF).

\begin{tabular}{|c|c|c|c|c|c|c|}
\hline & $M$ & $D T$ & Asimetría & Curtosis & PRE & CMC \\
\hline $\begin{array}{l}\text { Habla continuamente y no permite que } \\
\text { realicemos aportaciones en clase. }\end{array}$ & 4,43 & 0,93 & 0,96 & $-0,32$ & 0,44 & 0,20 \\
\hline $\begin{array}{l}\text { Explica muy poco las actividades que nos } \\
\text { plantea y no da alternativas de cómo } \\
\text { realizarla. }\end{array}$ & 4,10 & 1,21 & 1,69 & 1,86 & 0,41 & 0,17 \\
\hline $\begin{array}{l}\text { Coloca las fechas límite de entrega de } \\
\text { trabajosy tareas sin contar con nosotros. }\end{array}$ & 4,00 & 1,20 & 0,80 & $-0,74$ & 0,41 & 0,17 \\
\hline $\begin{array}{l}\text { Cuando no nos esforzamos en hacer las } \\
\text { cosas como él dice, nos hace menos caso. }\end{array}$ & 4,23 & 1,12 & 0,74 & $-0,94$ & 0,40 & 0,16 \\
\hline $\begin{array}{l}\text { Si está enfadado por algo, nos presta menos } \\
\text { atención. }\end{array}$ & 3,70 & 1,30 & 0,85 & $-0,69$ & 0,48 & 0,23 \\
\hline $\begin{array}{l}\text { Nos indica qué deberíamos hacer en nuestro } \\
\text { tiempo libre para que lo dediquemos a lo } \\
\text { que él quiere. }\end{array}$ & 2,95 & 1,48 & 0,78 & $-0,87$ & 0,33 & 0,11 \\
\hline $\begin{array}{l}\text { Cuando algo no sale como quiere utiliza } \\
\text { expresiones como: hazlo como yo te he } \\
\text { enseñado. }\end{array}$ & 3,89 & 1,42 & $-0,15$ & $-1,38$ & 0,35 & 0,12 \\
\hline $\begin{array}{l}\text { Suele decirnos que si hacemos las cosas } \\
\text { como él indica tendremos recompensas. }\end{array}$ & 2,99 & 1,39 & 0,28 & $-1,33$ & 0,36 & 0,130 \\
\hline $\begin{array}{l}\text { Nos presiona para que realicemos las } \\
\text { actividades como él dice. }\end{array}$ & 3,70 & 1,32 & 0,60 & $-1,74$ & 0,58 & 0,33 \\
\hline
\end{tabular}

Nota: PRE: Pesos de regresión, CMC: Correlación múltiple al cuadrado. 


\section{Resultados}

\section{Análisis descriptivos y de correlaciones bi- variadas}

El apoyo a la autonomía presentó una media de 3,82, el estilo controlador una media de 2,31, el feedback general positivo percibido de 3,94, el conocimiento del rendimiento percibido de 3,81, el feedback no verbal positivo percibido de 3,08 y el feedback no verbal negativo percibido de 2,36. Respecto al análisis de correlaciones bivariadas, el apoyo a la autonomía correlacionó positivamente con el feedback general positivo percibido, con el conocimiento del rendimiento percibido y con el feedback no verbal positivo general, mientras que correlacionó negativamente con el estilo controlador y el feedback no verbal negativo general. El estilo controlador correlacionó positivamente con el feedback no verbal negativo general y negativamente con el feedback general positivo percibido. El feedback general positivo percibido correlacionó positivamente con el conocimiento del rendimiento percibido $y$ el feedback no verbal positivo general, y obtuvo una correlación negativa con el feedback no verbal negativo general. El conocimiento del rendimiento percibido correlacionó positivamente con el feedback no verbal positivo general (Tabla 3).

\section{Tabla 3}

Análisis descriptivo y de correlaciones de todas las variables.

\begin{tabular}{|c|c|c|c|c|c|c|c|c|c|}
\hline \multicolumn{2}{|c|}{ Variables } & \multirow{2}{*}{$\frac{M}{3,82}$} & \multirow{2}{*}{$\begin{array}{c}D T \\
0,71\end{array}$} & \multirow[t]{2}{*}{1} & \multirow{2}{*}{$\frac{\mathbf{2}}{-0,37^{* *}}$} & \multirow{2}{*}{$\frac{3}{0,53^{* *}}$} & \multirow{2}{*}{$\frac{4}{0,48^{* *}}$} & \multirow{2}{*}{$\frac{\mathbf{5}}{0,38^{* *}}$} & \multirow{2}{*}{$\frac{6}{-0,22^{* *}}$} \\
\hline 1. & Apoyo a la autonomía & & & & & & & & \\
\hline 2. & Estilo controlador & 2,31 & 0,74 & & - & $-0,12^{*}$ & 0,08 & 0,01 & $0,36 * *$ \\
\hline 3. & $\begin{array}{l}\text { Feedback general positivo } \\
\text { percibido }\end{array}$ & 3,94 & 0,97 & & & - & $0,50^{* *}$ & $0,53^{* *}$ & $-0,18^{* *}$ \\
\hline 4. & $\begin{array}{l}\text { Conocimiento del rendimiento } \\
\text { percibido }\end{array}$ & 3,81 & 0,78 & & & & - & $0,36^{* *}$ & $-0,07$ \\
\hline 5. & $\begin{array}{l}\text { Feedback no verbal positivo } \\
\text { general }\end{array}$ & 3,08 & 0,91 & & & & & - & 0,09 \\
\hline 6. & $\begin{array}{l}\text { Feedback no verbal negativo } \\
\text { general }\end{array}$ & 2,36 & 1,12 & & & & & & - \\
\hline
\end{tabular}


Moreno-Murcia, J., Huéscar, E., Andrés-Fabra,J., \& Sánchez-Latorre, F. (2020). Adaptación y validación de los cuestionarios de apoyo a la autonomía y estilo controlador a la educación física: relación con el feedback.

Revista Ciencias de la Actividad Física UCM, N²1(1), enero-junio, 1-16. DOI: http://doi.org/10.29035/rcaf.21.1.3

\section{Modelo de regresión lineal}

Respecto al apoyo a la autonomía (Tabla 4), el análisis de regresión lineal mostró que el feedback no verbal negativo general predijo negativamente el apoyo a la autonomía, junto con el feedback general positivo percibido, el conocimiento del rendimiento percibido y el feedback no verbal positivo general que predijeron positivamente el apoyo a la autonomía en un 38\% de varianza explicada.
En cuanto al estilo controlador, el feedback no verbal negativo general predijo negativamente el apoyo a la autonomía, junto con el feedback general positivo percibido y el conocimiento del rendimiento percibido que predijeron positivamente el apoyo a la autonomía en un 13\% de varianza explicada.

\section{Tabla 4}

Análisis de Regresión lineal de predicción de la escala PTF-R sobre el Apoyo a la autonomía y el Estilo controlador.

\begin{tabular}{lcccc}
\hline & $B$ & $S E B$ & $\beta$ & $\Delta R^{2}$ \\
\hline Apoyo a la autonomía & 1,95 & 0,18 & & $0,38^{* *}$ \\
Feedback general positivo percibido & 0,21 & 0,04 & $0,29^{* *}$ & \\
Conocimiento del rendimiento percibido & 0,24 & 0,04 & $0,26^{* *}$ & \\
Feedback no verbal positivo general & 0,11 & 0,04 & $0,15^{*}$ & \\
Feedback no verbal negativo general & $-0,10$ & 0,28 & $-0,17^{* *}$ & \\
\hline Estilo controlador & 18,21 & 2,29 & & $0,13^{* *}$ \\
Feedback general positivo percibido & $-0,31$ & 0,17 & $-0,11^{*}$ & \\
Conocimiento del rendimiento percibido & 0,50 & 0,14 & $0,20^{* *}$ & \\
Feedback no verbal positivo general & 0,01 & 0,12 & 0,00 & \\
Feedback no verbal negativo general & 0,70 & 0,11 & $0,37^{* *}$ & \\
\hline
\end{tabular}

\section{Discusión}

Según el informe EURYDICE (2008) sobre autonomía y responsabilidades de los docentes en Europa, en la mayoría de los países europeos se han asignado nuevas responsabilidades al profesorado como resultado de la creciente autonomía de los centros escolares, esperando que este hecho sirva para desarrollar su creatividad y capacidad de innovación en pro de las necesidades de los estudiantes. Ante esta preocupación, este trabajo ha tenido como objetivo el diseño y validación al contexto de la educación física de las escalas EAA-EF (Escala de Apoyo a la Autonomía en Educación Física) y EEC-EF (Escala de Estilo Controlador en Educación Física) del docente en clases de educación física, así como comprobar la relación del feedback percibido (general positivo, conocimiento del rendimiento, no verbal positivo general y no verbal negativo general) sobre los dos estilos interpersonales docentes. 
Para determinar la estructura factorial de los instrumentos se han analizado sus propiedades psicométricas, revelando unos índices de ajuste aceptables del modelo de un factor cada una, quedando compuestas por 11 ítems la escala EAA-EF, y 9 ítems la escala EEC-EF. Ambas escalas mostraron también una adecuada fiabilidad. No obstante, la validación de estas escalas debe asistir a un proceso continuado en el futuro, por lo que serían necesarios más estudios que trataran de testar los resultados obtenidos con diferentes muestras y generando nuevos análisis estadísticos.

Como se planteaba en las hipótesis, el estilo docente percibido de apoyo a la autonomía correlacionó positivamente con el feedback general positivo percibido, con el conocimiento del rendimiento percibido y con el feedback no verbal positivo general, mientras que correlacionó negativamente con el estilo controlador y el feedback no verbal negativo general. Estudios previos ya han demostrado que la autonomía percibida por el alumnado de educación física se relacionaba con estos tres tipos de feedback (Huéscar \& Moreno-Murcia, 2012), incluso la existencia de relaciones positivas entre ellos (Koka \& Hein, 2003). Estos resultados apuntan a que determinadas estrategias de feedback pueden estar ejerciendo implicaciones diferentes en el apoyo a la autonomía entendido por el estudiante. Concretamente, el feedback de carácter positivo (verbal y no verbal) como el referido al chequeo periódico de las ejecuciones de los estudiantes (conocimiento del rendimiento) de carácter constructivo facilitan climas de aula orientados a la tarea (Viciana, Cervelló, Ramírez, San-Matías, \& Requena, 2003), evitando barreras de la comunicación y promoviendo finalmente la motivación intrínseca del alumnado (Moreno-Murcia et al., 2013). Estos resultados también van en la línea de la recomendación de la consistencia entre la comunicación verbal y no verbal en el aula (Yukelson, 1998) insistiendo en el papel de las orientaciones comunicativas del docente de educación física por su incidencia sobre la autonomía de los estudiantes (Nicaise, Cogerino, Bois, \& Amorós, 2006). No obstante, los resultados del estudio apuntan, además, que el tipo de feedback del conocimiento del rendimiento percibido ha de venir acompañado de las tipologías de feedback positivo para acercarse al estilo de apoyo a la autonomía del estudiante, mientras que por sí solo podría darse también dentro del estilo controlador. Un docente que impone su forma de hacer, pensar y comportarse a sus discentes es esperable que les proporcione periódicamente el balance de sus ejecuciones a los estudiantes para incitarles a cumplir los objetivos atribuidos.

Para seguir verificando la utilidad de ambas escalas resulta necesario continuar con futuras investigaciones revisando la construcción de sus ítems, así como respecto a la validez de criterio, relacionando ambos estilos con algunas de las variables implicadas en los mecanismos motivacionales contempladas en el marco teórico de la TAD. También sería interesante incluir las variables sexo y edad en docentes y discentes, así como la formación docente previa en metodologías activas. Finalmente, otra limitación del trabajo deriva de la propia construcción de los instrumentos al considerar las necesidades psicológicas básicas en un único constructo de forma conjunta. Pese a que esta elección ya ha sido considerada en anteriores trabajos (Standage y col., 2005) y que probablemente podría tratarse de una opción más sencilla hacia la optimización y gestión del estilo motivador docente, la conveniencia de promover estilos docentes que apoyen a la autonomía del estudiante también radica en la predicción ya demostrada (Standage et al., 2006) sobre la satisfacción de cada una de las necesidades de competencia, autonomía y relación con los demás, junto a la motivación intrínseca y otras variables como la persistencia de los estudiantes en clase. 
Moreno-Murcia, J., Huéscar, E., Andrés-Fabra,J., \& Sánchez-Latorre, F. (2020). Adaptación y validación de los cuestionarios de apoyo a la autonomía y estilo controlador a la educación física: relación con el feedback.

Revista Ciencias de la Actividad Física UCM, N²1(1), enero-junio, 1-16. DOI: http://doi.org/10.29035/rcaf.21.1.3

\section{Conclusiones}

Estos resultados aportan información relevante dada la necesidad de encontrar instrumentos que permitan favorecer no solamente climas adecuados en clases de educación física (Standage et al., 2006), sino que partan de discriminar qué actuaciones concretas que conforman ciertos patrones de interacción docente son los que pueden redundar en el incremento de la motivación positiva de los estudiantes. En esta línea, sería conveniente que nuevos trabajos abordaran el estudio de los estilos de interacción docente-discente a través de diseños experimentales tratando de encontrar relaciones causa-efecto entre un estilo concreto (apoyo a la autonomía vs. controlador), tipo de feedback, satisfacción de las necesidades psicológicas básicas y otras variables adaptativas (como la satisfacción con la vida o el compromiso deportivo).

\section{Agradecimientos}

Este documento ha sido financiado en parte por el proyecto de investigación DEP2017-83441-R titulado "Más allá del control: Consecuencias del fomento del apoyo a la autonomía en docentes y estudiantes en clases de educación física", perteneciente a la convocatoria 2017 de Proyectos I+D+l, del Programa Estatal de Investigación, Desarrollo e Innovación orientada a los Retos de la Sociedad del Ministerio de Economía, Industria y Competitividad (España).

\section{REFERENCIAS BIBLIOGRÁFICAS}

Abós, A., Sevil, J., Sanz, M., Aibar, A., \& GarcíaGonzález, L. (2016). El soporte de autonomía en Educación Física como medio de prevención de la oposición desafiante del alumnado. RICYDE. Revista Internacional de Ciencias del Deporte, 12(43), 65-78. Recuperado de http://dx.doi.org/10.5232/ricyde2016.04304

Aibar, A., Julián, J. A., Murillo, B., García-González, L., Estrada, S., \& Bois, J. (2015). Actividad física y apoyo a la autonomía: El rol del profesor de Educación Física. Revista de Psicología del Deporte, 24(1), 155-161. Recuperado de https://psycnet.apa.org/record/2015-15496-017

Azorín, F., \& Sánchez-Crespo, J. L. (1986). Métodos y aplicaciones del muestreo. Madrid: Alianza Universidad.

Bartholomew, K. J., Ntoumanis, N., \& Thøgersen-Ntoumani, C. (2010). The controlling interpersonal style in a coaching context: Development and initial validation of a psychometric scale. Journal of Sport y Exercise Psychology, 32(2), 193-216. DOI: 10.1123/jsep.32.2.193

Byrne, B. (2001). Structural Equation Modeling with EQS and EQS/Windows. United States: SAGE Publications, Inc.

Castillo, I., Tomás, I., Ntoumanis, N., Bartholomew, K., Duda, J. L., \& Balaguer, I. (2014). Psychometric properties of the Spanish version of the Controlling Coach Behaviors Scale in the sport context. Psicothema, 26(3), 409-414. DOI: 10.7334/psicothema2014.76 
Deci, E. L., \& Ryan, R. M. (2000). The 'What' and 'Why' of goal pursuits: Human needs and the self-determination of behaviour. Psychological Inquiry, 77(4), 227268. DOI: 10.1207/S15327965PLI1104_01

De Miguel, A., Marrero, R. J., Fumero, A., \& Carballeira, M. (2017). El papel de la personalidad y la inteligencia en la autoeficacia interpersonal. Revista Iberoamericana de Diagnóstico y Evaluación - Avaliação Psicológica. RIDEP, 44(2), 16-27. Recuperado de https://doi.org/10.21865/RIDEP44.2.02

Eurydice (2008). Autonomía y responsabilidades del profesorado en Europa. Bruselas: Eurydice.

Finney, S. J., \& DiStefano, C. (2006). Non-normal and Categorical data in structural equation modeling. En G. R. Hancock \& R. O. Mueller (Eds.), Structural equation modeling: a second course (pp. 269-314). Greenwich, Connecticut: Information Age Publishing

Gallagher, P., Yancy, W. S., Swartout, K., Denissen, J. J., Kuhnel, A., \& Voils, C. I. (2012). Age and sex differences in prospective effects of health goals and motivations on daily leisure-time physical activity. Preventive Medicine, 55(4), 322-324. DOI: 10.1016/j.ypmed.2012.07.017

Granero-Gallegos, A., Baena-Extremera, A., Pérez-Quero, F. J., Ortiz-Camacho, M. M., \& Bracho-Amador, C. (en prensa). Validación española del "Intention to partake in leisure-time physical activity". Retos. Nuevas tendencias en Educación Física, Deportes y Recreación, 26. Recuperado de https:// recyt.fecyt.es/index.php/retos/article/ view/34392
Haerens, L., Kirk, D., Cardon, G., De Bourdeaudhuij, I., \& Vansteenkiste, M. (2010). Motivational profiles for secondary school physical education and its relationship to the adoption of a physically active lifestyle among university students. European Physical Education Review, 16(2), 117-139. DOI: $10.1177 / 1356336 \times 10381304$

Hagger, M. S., Chatzisarantis, N. L. D., Hein, V., Pihu, M., Soós, I., \& Karsai, I. (2007). The perceived autonomy support scale for exercise settings (PASSES): Development, validity and cross-cultural invariance in young people. Psychology of Sport and Exercise, 8(5), 632653. Recuperado de http://dx.doi. org/10.1016/j.psychsport.2006.09.001

Huéscar, E., \& Moreno-Murcia, J. A., (2012). Relación del tipo de feed-back del docente con la percepción de autonomía del alumnado en clases de educación física. Infancia y Aprendizaje, 35(1), 87-98. DOI: $10.1174 / 021037012798977449$

Huéscar, E., \& Moreno-Murcia, J. A. (2017). Apoyo a la autonomía entre estudiantes, estrés percibido y miedo a la evaluación negativa: Relaciones con la satisfacción con la vida. Behavioral Psychology /Psicología Conductual. 25(3), 517-528.

Huéscar, E., Moreno-Murcia, J. A., \& Alías, A. (2017). Diseño y validación de una escala para medir el estilo controlador del estudiante de Educación Superior. Psychology, Society, y Education, 9(2), 239-249. DOI: 10.25115/psye. v9i2.696 
Moreno-Murcia, J., Huéscar, E., Andrés-Fabra,J., \& Sánchez-Latorre, F. (2020). Adaptación y validación de los cuestionarios de apoyo a la autonomía y estilo controlador a la educación física: relación con el feedback.

Revista Ciencias de la Actividad Física UCM, N²1(1), enero-junio, 1-16. DOI: http://doi.org/10.29035/rcaf.21.1.3

Koka, A., \& Hein, V. (2003). Perceptions of teacher's feedback and learning environment as predictors of intrinsic motivation in physical education. Psychology of Sport and Exercise, 4(4), 333-346. Recuperado de http://dx.doi.org/10.1016/S14690292(02)00012-2.

Koka, A., \& Hein, V. (2005). The effect of perceived teacher feed-back on intrinsic motivation in physical education. International Journal of Sport Psychology, 36(2), 91-106. Recuperado de http://kodu.ut.ee/ vello/1.pdf

Ley Orgánica 8/2013, de 9 de diciembre, para la mejora de la calidad educativa, (LOMCE). BOE, núm. 295, de 10 de diciembre de 2013, (97858-97921). Recuperado de http://www.edu.xunta.gal/centros/cpivianopequeno/system/files/LOMCE\%2OTexto\%20refundido\%20con\%2OLOE.pdf

Lim, B. S., \& Wang, C. K. (2009). Perceived autonomy support, behavioural regulations in physical education and physical activity intention. Psychology of Sport and Exercise, 10(1), 52-60. Recuperado de https://doi.org/10.1016/j. psychsport.2008.06.003

Moreno-Murcia, J. A., Huéscar, E., Peco, N., Alarcón, E., \& Cervelló, E. (2013). Relación del feed-back y las barreras de comunicación del docente con la motivación intrínseca de estudiantes adolescentes de educación física. Anales de Psicología, 29(1), 257-263. Recuperado de http://dx.doi. org/10.6018/analesps.29.1.161881
Moreno-Murcia, J. A., Huéscar, E., Pintado, R., \& Marzo, J. C. (2019). Diseño y validación de la Escala de Apoyo a la Autonomía en Educación Superior. Relación con la competencia laboral del discente. Revista Española de Orientación y Psicopedagogía, 30(1), 116-130. Recuperado de http://revistas.uned.es/index.php/reop/article/ view/25197

Moreno-Murcia, J. A., Pintado, R., Huéscar, E., \& Marzo, J. C. (2018). Estilo interpersonal controlador y percepción de competencia en educación superior. European Journal of Education and Psychology, 77(7), 33-45. Recuperado de https://www.redalyc.org/ pdf/1293/129353356003.pdf

Moreno-Murcia, J. A., Ruíz, M., Silveira, Y., \& Alias, A. (2017). Estimación del estudiante profundo a través de un modelo cognitivo-social. Profesorado. Revista de currículum y formación del profesorado, 27(3), 239-257. Recuperado de https://www.redalyc.org/ pdf/567/56752489012.pdf

Nicaise, V., Cogerino, G., Bois, J., \& Amorose, A. J. (2006). Students' perceptions of teacher feedback and physical competence in physical education classes: Gender effects. Journal of Teaching in Physical Education, 25(1), 36-57. DOI: https://doi.org/10.1123/ jtpe.25.1.36

Oguz, A. (2013). Developing a scale for learner autonomy support. Educational Sciences: Theory and Practice, 13(4), 2187-2194. DOI: 10.12738/ estp.2013.4.1870 
Öztürk, I. H. (2011). Curriculum reform and teacher autonomy in Turkey: The case of the history teaching. International Journal of Instruction. 4(2), 113-128.

PISA (2012). Results, Ready to Learn: Student's Engagement, Drive, and Self-Beliefs (Volume III). Paris: OECD Publishing. Recuperado de https:// www.oecd.org/pisa/keyfindings/pisa2012-results-volume-III.pdf

Reeve, J. (2009). Why teachers adopt a controlling motivating style toward students and how they can become more autonomy supportive. Educational Psychologist, 44(3), 159-175. Recuperado de http://dx.doi. org/10.1080/00461520903028990

Röder, B., \& Kleine, D. (2007). Selbstbestimmung/Autonomie. En M. Jerusalem, S. Drössler, D. Kleine, J. Klein-HeBling, W. Mittag, y B. Röder (Eds.), Förderung von Selbstwirksamkeitund SeIbstbestimmungim Unterricht. Berlin: Humboldt- Universitätzu Berlin.

Ryan, R. M., \& Deci, E. L. (2000). Self-determination theory and the facili- tation of intrinsic motivation, social development and well-being. American Psychologist, 55(7), 68-78. DOI: 10.1037/0003-066X.55.1.68

Sánchez-Oliva, D., Leo, F. M., Amado, D., Cuevas, R., \& García-Calvo, T. (2013). Desarrollo y validación del Cuestionario de Apoyo a las Necesidades Psicológicas Básicas en Educación Física. Motricidad. European Journal of Human Movement, 30, 53-71. Recuperado de https://www.redalyc.org/ pdf/2742/274228060005.pdf
Soenens, B., Park, S.Y., Vansteenkiste, M., \& Mouratidis, A. (2012). Perceived parental psychological control and adolescent depressive experiences: A cross-cultural study with Belgian and South-Korean adolescents. Journal of Adolescence, 35(2), 261,-272. Recuperado de https://doi.org/10.1016/j.adolescence.2011.05.001

Standage, M., Duda, J. L. \& Ntoumanis, N. (2005). A test of self-determination theory in school physical education. British Journal of Educational Psychology, 75(3),471433. Recuperado de https://doi. org/10.1348/000709904X22359

Standage, M., Duda, J. L., \& Ntoumanis, N. (2006). Students' motivational processes and their relationship to teacher ratings in school physical education: A self-determination theory approach. Research Quarterly for Exercise and Sport, 77(1), 100-110. Recuperado de https://doi.org/10.1080/0 2701367.2006 .10599336

TALIS (2013). Estudio internacional de la enseñanza y el aprendizaje. Análisis secundario. Informe español. MECD.

Teixeira, M. O., \& Laranjeira, M. (2018). Interesses e Perceções de Competência na Infância: Um Estudo Exploratório no Contexto Português. Revista Iberoamericana de Diagnóstico y Evaluación-e Avaliação Psicológica, 2(47), 37-52. Recuperado de https://www.redalyc.org/jatsRepo/4596/459655209004/459655209004. pdf

Ureña, N., Alarcón, F., \& Ureña, F. (2009). La realidad de los deportes colec- tivos en la Enseñanza Secundaria. Cómo planifican e intervienen los profesores de Murcia. Retos. Nuevas Tendencias en Educación Física, Deportes y Recreación, 16, 9-15. 
Moreno-Murcia, J., Huéscar, E., Andrés-Fabra,J., \& Sánchez-Latorre, F. (2020). Adaptación y validación de los cuestionarios de apoyo a la autonomía y estilo controlador a la educación física: relación con el feedback.

Revista Ciencias de la Actividad Física UCM, N²1(1), enero-junio, 1-16. DOI: http://doi.org/10.29035/rcaf.21.1.3

Valdivia, J. (2011). La retroalimentación correctiva y de reforzamiento en clases de educación física. TRANCES. Transmisión del Conocimiento Educativo y de la Salud, 3(5), 687-694.

Vallejo, G., \& Plested, M. C. (2008). Tipos de comunicación en interacciones deportivas. Ikala. Revista de Lenguajey Cultura, 13(19), 43-61. Recuperado de http://hdl.handle.net/10495/2983

Vallerand, R. J. (1997). Toward a hierarchical model of intrinsic and extrinsic motivation. En Zanna, M. P. (Ed.), Advances in experimental social psychology (pp. 271-360). San Diego: Academic Press.

Viciana, J., Cervelló, E., Ramírez, J., San-Matías, J., \& Requena, B. (2003). Influencia del feedback positivo y negativo en alumnos de secundaria sobre el clima ego-tarea percibido, la valoración de la educación física y la preferencia en la complejidad de las tareas de clase. Motricidad, 10, 99-116. Recuperado de https://www.redalyc.org/ pdf/2742/274220877005.pdf

Williams, G. C., \& Deci, E. L. (1996). Internalization of biopsychosocial values by medical students: A test of self-determination theory. Journal of Personality and Social Psychology, 70, 767-779. DOI: 10.1037//0022-3514.70.4.767

Yukelson, D. (1998). Communication effectively. En Williams, J. M. (Ed.), Applied sport psychology: Personal growth to peak performance, 3rd ed., (pp. 142157). Mountain View: Mayfield.

\section{Dirección para correspondencia}

Elisa Huéscar Hernández

Departamento de Psicología de la Salud Universidad Miguel Hernández de Elche Edificio Altamira

Avenida de la Universidad, s/n. 03202 Elche, Alicante, España.

Tel: 966658525

ORCID: https://orcid.org/0000-0002-2713-4506

Contacto:

ehuescar@umh.es

Recibido: 27-10-2019

Aceptado: 23-03-2020 